\title{
RESULTS ON AN INTEGRAL INEQUALITY OF THE OPIAL- TYPE
}

K. RAUF AND Y. O. ANTHONIO

(Received 19 October 2016; Revision Accepted 3 November 2016)

\section{ABSTRACT}

We obtain integral inequalities which are Opial-type inequalities, mainly by using Jensen's inequality for the case of convex function.

KEYWORDS: Integral inequalities, Opial's inequality, Jensen's inequality and convex functions.

2010 Subject Classification: 15A31.

\section{INTRODUCTION}

Opial ([8])established the following interesting integral inequality:

Let $x(t) \in C^{\prime}[0, b]$ be such $x(0)=x(b)=0$ and $x(t)>0$ in $(0, \mathrm{~b})$, then

$$
\int_{a}^{b}\left|x(t) x^{\prime}(t)\right| d t \leq \frac{b}{4} \int_{a}^{b}\left(x^{\prime}(t)\right)^{2} d t
$$

where $\frac{b}{4}$ in the best possible constant.

In 1967 Maroni [5] obtained a generalized Opial's inequality by using $H \ddot{o}$ Iders inequality with indices $\mu$ and $v$. The result obtained is the following:

\section{Theorem 1:}

Let $\mathrm{p}(\mathrm{t})$ be positive and continuous on $[\tau, \alpha]$ with $\int_{\alpha}^{\tau} p^{1-\mu}(t) d t<\infty$, where $\mu>1, \mathrm{x}(\mathrm{t})$ be absolutely function on $[\alpha, \tau]$ and $x(0)=0$. Then, the following inequality holds.

$$
\int_{\alpha}^{\tau}\left|x(t) x^{\prime}(t)\right| d t \leq \frac{1}{2}\left(\int_{\alpha}^{\tau} p^{1-\mu}(t) d t\right)^{\frac{2}{\mu}}\left(\int_{\alpha}^{\tau} p(t)\left|x^{\prime}(t)\right|^{\nu} d t\right)^{\frac{2}{v}}
$$

where $\frac{1}{\mu}+\frac{1}{v}=1$. Equality holds in (2)iff $c \int_{\alpha}^{\tau} p^{1-\mu}(s) d s$.

K. Rauf, Department of Mathematics, University of Ilorin, Ilorin, Nigeria.

Y.O. Anthonio, Department of Mathematics, University of Ilorin, Ilorin, Nigeria. 
Calvert [2] also established the following result:

Theorem 2: [2] Assume that

(i) $\quad \mathrm{x}(\mathrm{t})$ is absolutely continuous in $[\alpha, \tau]$ and $x(\alpha)=0$

(ii) $f(t)$ is continuous, complex-valued, defined in the range of $x(t)$ and for all real for $t$ of the form $t(s)=\int_{\alpha}^{s}\left|x^{\prime}(u)\right| d u: f(|t|)$ for all $\mathrm{t}$ and $\mathrm{f}(\mathrm{t})$ is real $t>0$ and is increasing there,

(iii) $\quad \mathrm{p}(\mathrm{t})$ is positive, continuous and $\int_{\alpha}^{\tau} p^{1-\mu}(t) d t<\infty$, where $\frac{1}{\mu}+\frac{1}{v}=1$. Then the following inequality holds.

$$
\int_{\alpha}^{\tau}\left|f(x(t)) x^{\prime}(t)\right| d t \leq F\left[\left(\int_{\alpha}^{\tau} p^{1-\mu}(t) d t\right)^{\frac{1}{\mu}}\left(\int_{\alpha}^{\tau} p(t)\left|x^{\prime}(t)\right|^{\nu} d t\right)^{\frac{1}{v}}\right]
$$

where $F(t)=\int_{0}^{t} f(t) d s, t>0$. Equality holds in (3) iff $x(t)=\int_{\alpha}^{t} p^{1-\mu}(s) d s$.

The aim of this paper is to generalize Maroni and Calvert results using Jensen's inequality.

\section{Some Adaptations of Jensen's inequalities :}

Let $\varphi$ be continuous and convex function and let $\mathrm{h}(\mathrm{s}, \mathrm{t})$ be a non negative function and $\lambda$ be non decreasing function. Let $-\infty \leq \xi(t) \leq \eta(t)<\infty$ and suppose $\varphi$ has a continuous inverse $\varphi^{-1}$ (which is necessarily concave). Then,

$$
\varphi^{-1}\left(\left|\frac{\int_{\xi(t)}^{\eta(t)} h(s, t) d \lambda(s)}{\int_{\xi(t)}^{\eta(t)} d \lambda(s)}\right|\right) \leq\left(\frac{\int_{\xi(t)}^{\eta(t)}(\varphi)^{-1}(|h(s, t)|) d \lambda(s)}{\int_{\xi(t)}^{\eta(t)} d \lambda(s)}\right)
$$

with the inequality reversed if $\varphi$ is concave. The inequality (4) above is known as Jensen's inequality for convex function. Setting $\varphi(u)=u^{l-1}, \xi(t)=0$, and $\eta(t)=t$ in (4), then we obtain

$$
(f(t))^{\zeta}=\left(f\left(\left|\frac{\int_{0}^{t} h(s, t) d \lambda(s)}{\int_{0}^{t} d \lambda(s)}\right|\right)\right)^{\frac{1}{\zeta}} \leq\left(\frac{\int_{0}^{t}(|h(s, t)|)^{\frac{1}{l}} d \lambda(s)}{\int_{0}^{t} d \lambda(s)}\right) .
$$

\section{MAIN RESULT:}

Before stating our main result in this section, we shall need the following useful Lemma:

\section{Lemma 1:}

Let $x(t), \lambda(t)$ and $f(u)$ be absolutely continuous and non decreasing functions on $[a, b]$ for $0 \leq a \leq b<\infty$ with $f(t)>0$. Let $\alpha, \beta, k$ and $\varepsilon$ be real numbers such that $\delta, \geq 0, \varepsilon \geq 0$ and also Let $P(x)$, and $R(t)$ be non negative and measurable function on $[a, b]$ such that

$$
\left|x^{\prime}(t)\right| \times f\left(\left|\int_{0}^{t} x^{\prime}(t) R(t) d \lambda(t)\right|\right) \leq \lambda(t)^{1-\delta-\varepsilon} y(t)^{\delta} R(t)^{-1} \lambda^{\prime}(t)^{-1} y^{\prime}(t)
$$

Then, the following inequality holds:

$$
\int_{a}^{b}\left|x^{\prime}(t)\right| \times f\left(\int_{0}^{t}\left|x^{\prime}(t)\right| d t\right) \leq \int_{a}^{b} y(t)^{\varepsilon} d y^{\prime}(t) .
$$




\section{Proof:}

Setting $h(s, t)=x^{\prime}(t) R(t)$ in (5), we have

$$
(f(t))^{\varepsilon}=\left(f\left(\left|\frac{\int_{0}^{t} x^{\prime}(t) R(t) d \lambda(t)}{\int_{0}^{t} d \lambda(t)}\right|\right)\right)^{\frac{1}{\varepsilon}} \leq\left(\frac{\int_{0}^{t}\left(\left|x^{\prime}(t) R(t)\right|\right)^{\frac{1}{l}} d \lambda(t)}{\int_{0}^{t} d \lambda(t)}\right) .
$$

By setting $f(\lambda(t))=\lambda(t)^{1-\delta}$ in (8) yeilds

$$
\frac{f\left(\left|\int_{0}^{t} x^{\prime}(t) R(t) d \lambda(t)\right|\right)}{\lambda(t)^{1-\delta}} \leq \frac{\left(\int_{0}^{t} f\left(\left|x^{\prime}(t) R(t)\right|\right)^{\frac{1}{1-\delta}} d \lambda(t)\right)^{\varepsilon}}{\lambda(t)^{\varepsilon}} .
$$

Hence

$$
f\left(\left|\int_{0}^{t} x^{\prime}(t) R(t) d \lambda(t)\right|\right) \leq \lambda(t)^{1-\delta-\varepsilon}\left(\int_{0}^{t} f\left(\left|x^{\prime}(t) R(t)\right|\right)^{\frac{1}{1-\delta}} d \lambda(t)\right)^{\varepsilon}=\lambda(t)^{1-\delta-\varepsilon} y(t)^{\varepsilon} .
$$

Now let

$$
y(t)=\int_{0}^{t} f\left(\left|x^{\prime}(t) R(t)\right|\right)^{\frac{1}{1-\delta}} \lambda^{\prime}(t)
$$

then

$$
y^{\prime}(t)=f\left(\left|x^{\prime}(t) R(t)\right|\right)^{\frac{1}{1-\delta}} \lambda^{\prime}(t)
$$

That is,

$$
y^{\prime}(t)^{1-\delta}=f\left(\left|x^{\prime}(t) R(t)\right|\right) \lambda^{\prime}(t)^{1-\delta} .
$$

Using the fact that $f(u)=u^{1-\delta}$ to have

$$
\begin{aligned}
& y^{\prime}(t)^{1-\delta}=\left|x^{\prime}(t)\right|^{1-\delta} R(t)^{1-\delta} \lambda^{\prime}(t)^{1-\delta} . \\
& \left|x^{\prime}(t)\right|=R(t)^{-1} \lambda^{\prime}(t)^{-1} y^{\prime}(t),
\end{aligned}
$$

Combining both (10) and (15) to yeilds, inequality (6) and the proof is complete.

$$
\left|x^{\prime}(t)\right| \times f\left(\left|\int_{0}^{t} x^{\prime}(t) R(t) d \lambda(t)\right|\right) \leq \lambda(t)^{1-\delta-\varepsilon} y(t)^{\delta} R(t)^{-1} \lambda^{\prime}(t)^{-1} y^{\prime}(t)
$$

\section{Remarks 1:}

By setting $f(u)=u^{1-\delta}, R(t)=P(t)^{-\frac{1}{k-1}}, \lambda^{\prime}(t)^{\frac{1}{k-1}}, 1-\delta=\varepsilon \quad$ in Lemma 1 yeilds

$$
\left|x^{\prime}(t)\right| \times f\left(\left|\int_{0}^{t} x^{\prime}(t) P(t)^{-\frac{1}{k-1}} P(t)^{\frac{1}{k-1}}\right| d t\right) \leq \lambda(t)^{1-\delta-\varepsilon} y(t)^{\varepsilon} \times P(t)^{-\frac{1}{k-1}} P(t)^{\frac{1}{k-1}} y^{\prime}(t) .
$$

Integrating both sides of inequality $(16)$ over $[a, b]$ with the respect to $t$, to get 
154

K. RAUF AND Y. O. ANTHONIO

$$
\int_{a}^{b}\left|x^{\prime}(t)\right| \times f\left(\int_{0}^{t}\left|x^{\prime}(t)\right| d t\right) \leq \int_{a}^{b} y(t)^{\varepsilon} y^{\prime}(t) d t
$$

That is,

$$
\begin{aligned}
& \int_{a}^{b}\left|x^{\prime}(t)\right| \times f\left(\int_{0}^{t}\left|x^{\prime}(t)\right| d t\right) \leq \int_{a}^{b} y(t)^{\varepsilon} d y^{\prime}(t) . \\
& \int_{a}^{b}\left|x^{\prime}(t)\right| \times f\left(\int_{0}^{t}\left|x^{\prime}(t)\right| d t\right) \leq \frac{y(t)^{\varepsilon+1}}{\varepsilon+1}
\end{aligned}
$$

Setting $\int_{0}^{t}\left|x^{\prime}(t)\right| d t=x(t)$ By using $\mathrm{H} \ddot{o}$ Ides inequality with $\alpha$ and $\beta$ we obtain

$$
\begin{aligned}
& \frac{1}{\varepsilon+1} y(b)^{\varepsilon+1}=\frac{1}{\varepsilon+1}\left(\int_{a}^{b}\left|x^{\prime}(t)\right| d t\right)^{\varepsilon+1}=\frac{1}{\varepsilon+1}\left(\int_{a}^{b} R^{-\frac{\varepsilon+1}{\alpha}}(t) R^{\frac{\varepsilon+1}{\beta}}\left|x^{\prime}(t)\right|(t) d t\right)^{\varepsilon+1} \\
& \leq \frac{1}{\varepsilon+1}\left(\int_{a}^{b} R^{1-\alpha}(t) d t\right)^{\frac{\varepsilon+1}{\alpha}}\left(\int_{a}^{b} R(t)\left|x^{\prime}(t)\right|^{\beta} d t\right)^{\frac{\varepsilon+1}{\beta}}
\end{aligned}
$$

Combining inequality (19) and (20) to obtain the Opial's Type inequality of the following

$$
\int_{a}^{b} x^{\prime}(t) f(x(t)) d t \leq \frac{1}{\varepsilon+1}\left(\int_{a}^{b} R^{1-\alpha}(t) d t\right)^{\frac{\varepsilon+1}{\alpha}}\left(\int_{a}^{b} R(t)\left|x^{\prime}(t)\right|^{\beta} d t\right)^{\frac{\varepsilon+1}{\beta}}
$$

which gives

$$
\int_{a}^{b}\left|x^{\prime}(t) x(t)^{1-\delta}\right| d t \leq \frac{1}{\varepsilon+1}\left(\int_{a}^{b} R^{1-\alpha}(t) d t\right)^{\frac{\varepsilon+1}{\alpha}}\left(\int_{a}^{b} R(t)\left|x^{\prime}(t)\right|^{\beta} d t\right)^{\frac{\varepsilon+1}{\beta}}
$$

Remark 2:

For $\varepsilon=0$ in inequality (22) yeilds

$$
\int_{a}^{b}\left|x^{\prime}(t) x(t)^{1-\delta}\right| d t \leq\left(\int_{a}^{b} R^{1-\alpha}(t) d t\right)^{\frac{1}{\alpha}}\left(\int_{a}^{b} R(t)\left|x^{\prime}(t)\right|^{\beta} d t\right)^{\frac{1}{\beta}}
$$

Putting $\varepsilon=1$, and $\delta=0$ in inequality (22) reduces to

$$
\int_{a}^{b}\left|x^{\prime}(t) x(t)\right| d t \leq \frac{1}{2}\left(\int_{a}^{b} R^{1-\alpha}(t) d t\right)^{\frac{2}{\alpha}}\left(\int_{a}^{b} R(t)\left|x^{\prime}(t)\right|^{\beta} d t\right)^{\frac{2}{\beta}}
$$

which generalizes inequality (2).

If $\varepsilon=1$ and $\alpha=0$ in inequality (22) yeilds

$$
\int_{a}^{b}\left|x^{\prime}(t) x(t)\right| d t \leq \frac{1}{2}\left(\int_{a}^{b} R(t)\left|x^{\prime}(t)\right|^{\beta} d t\right)^{\frac{2}{\beta}}
$$

If $\varepsilon=0$ in inequality (22) becomes

$$
\int_{a}^{b}\left|x^{\prime}(t) x(t)^{1-\delta}\right| d t \leq\left(\int_{a}^{b} R^{1-\alpha}(t) d t\right)^{\frac{1}{\alpha}}\left(\int_{a}^{b} R(t)\left|x^{\prime}(t)\right|^{\beta} d t\right)^{\frac{1}{\beta}}
$$

In inequality (18) if we set $1-\delta=\varepsilon$ becomes

$$
\int_{a}^{b}\left|x^{\prime}(t)\right| \times f\left(\int_{0}^{t}\left|x^{\prime}(t)\right| d t\right) \leq \int_{a}^{b} y(t)^{1-\delta} y^{\prime}(t) d t .
$$


That is,

$$
\int_{a}^{b}\left|x^{\prime}(t)\right| \times f\left(\int_{0}^{t}\left|x^{\prime}(t)\right| d t\right) \leq \int_{a}^{b} f(y(t)) d y^{\prime}(t) .
$$

Getting $\int_{0}^{t}\left|x^{\prime}(t)\right| d t=x(t)$

Using $\mathrm{H} \ddot{o}$ Ider's inequality with indices $\alpha$ and $\beta$, we have

$$
\int_{a}^{b} x^{\prime}(t) d t=\int_{a}^{b} R^{-\frac{1}{\alpha}}(t) R^{\frac{1}{\beta}}\left|x^{\prime}(t)\right|(t) d t \leq\left(\int_{a}^{b} R^{1-\alpha}(t) d t\right)^{\frac{1}{\alpha}}\left(\int_{a}^{b} R(t)\left|x^{\prime}(t)\right|^{\beta} d t\right)^{\frac{1}{\beta}}
$$

Combining (28) and (29) to obtain the following inequality

$$
\int_{a}^{b}\left|x^{\prime}(t)\right| \times f\left(\int_{0}^{t}\left|x^{\prime}(t)\right| d t\right) \leq f\left(\left(\int_{a}^{b} R^{1-\alpha}(t) d t\right)^{\frac{1}{\alpha}}\left(\int_{a}^{b} R(t)\left|x^{\prime}(t)\right|^{\beta} d t\right)^{\frac{1}{\beta}}\right)
$$

that is, inequality that generalizes inequality (3)

$$
\int_{a}^{b}\left|x^{\prime}(t) f(x(t))\right| d t \leq f\left(\left(\int_{a}^{b} R^{1-\alpha}(t) d t\right)^{\frac{1}{\alpha}}\left(\int_{a}^{b} R(t)\left|x^{\prime}(t)\right|^{\beta} d t\right)^{\frac{1}{\beta}}\right)
$$

Similarly, we need the following Lemma to obtain a new Opial's type inequality using Jensen's inequality for the case of convex function.

\section{Lemma 2 :}

Let $x(t), \lambda(t), f(u), R(t), l, k$ and $o \geq 0$ and $\rho \geq 0$ be as in Lemma 1 such that

$$
\left|x^{\prime}(t)\right| \times f\left(\left|\int_{0}^{t} x^{\prime}(t) R(t) d \lambda(t)\right|\right) \leq y^{\prime}(t) R(t)^{-1} \lambda^{\prime}(t)^{-1} y(t)^{\delta-1}
$$

Then, the following inequality holds:

$$
\left|x^{\prime}(t)\right| f\left(\int_{0}^{t}\left|x^{\prime}(t)\right| d t\right) \leq y(t)^{\delta-1} y^{\prime}(t) \text {. }
$$

\section{Proof:}

The proof is similar to the proof of Lemma 1.

Since $f(u)=u^{\delta-1}$, inequality (8) becomes

$$
\begin{aligned}
& \left.\left(f\left(\mid \frac{\int_{0}^{t} x^{\prime}(t) R(t) d \lambda(t)}{\int_{0}^{t} d \lambda(t)}\right)\right)\right)^{\frac{1}{(\delta-1)}} \leq\left(\frac{\int_{0}^{t} f\left(\left|x^{\prime}(t) R(t)\right|\right)^{\frac{1}{\delta-1}} d \lambda(t)}{\int_{0}^{t} d \lambda(t)}\right) \\
& f\left(\left|\frac{\int_{0}^{t} x^{\prime}(t) R(t) d \lambda(t)}{\int_{0}^{t} d \lambda(t)}\right|\right) \leq\left(\frac{\int_{0}^{t} f\left(\left|x^{\prime}(t) R(t)\right|\right)^{\frac{1}{\delta-1}} d \lambda(t)}{\int_{0}^{t} d \lambda(t)}\right)^{\delta-1} .
\end{aligned}
$$




$$
\begin{aligned}
& f\left(\left|\int_{0}^{t} x^{\prime}(t) R(t) d \lambda(t)\right|\right) \leq \lambda(t)^{\delta-1+1-\delta}\left(\int_{0}^{t} f\left(\left|x^{\prime}(t) R(t)\right|\right)^{\frac{1}{\delta-1}} d \lambda(t)\right)^{\delta-1} . \\
& f\left(\left|\int_{0}^{t} x^{\prime}(t) R(t) d \lambda(t)\right|\right) \leq\left(\int_{0}^{t} f\left(\left|x^{\prime}(t) R(t)\right|\right)^{\frac{1}{\delta-1}} d \lambda(t)\right)^{\delta-1} . \\
& y^{\prime}(t)=\left|x^{\prime}(t)\right| R(t) \lambda^{\prime}(t) \\
& y^{\prime}(t) R(t)^{-1} \lambda^{\prime}(t)^{-1}=\left|x^{\prime}(t)\right|
\end{aligned}
$$

Combining (37) and (39) to have

$$
\left|x^{\prime}(t)\right| \times f\left(\left|\int_{0}^{t} x^{\prime}(t) R(t) d \lambda(t)\right|\right) \leq y^{\prime}(t) R(t)^{-1} \lambda^{\prime}(t)^{-1} y(t)^{\delta-1}
$$

This completes the proof of the Lemma.

Consider all conditions of Remark 1

$$
\begin{aligned}
& \left|x^{\prime}(t)\right| \times f\left(\left|\int_{0}^{t} x^{\prime}(t) P(t)^{-\frac{1}{k-1}} P(t)^{\frac{1}{k-1}}\right|\right) \leq y^{\prime}(t) y(t)^{\delta-1} P(t)^{-\frac{1}{k-1}} P(t)^{\frac{1}{k-1}} y^{\prime}(t) . \\
& \left|x^{\prime}(t)\right| f\left(\int_{0}^{t}\left|x^{\prime}(t)\right| d t\right) \leq y(t)^{\delta-1} y^{\prime}(t)
\end{aligned}
$$

Then, putting $\int_{0}^{t}\left|x^{\prime}(t)\right| d t=x(t)$ and integrate both side of inequality above, over $[a, b]$ with the respect to $t$, yeilds

$$
\begin{aligned}
& \int_{a}^{b}\left|x^{\prime}(t) x(t)^{\delta-1}\right| d t \leq \int_{a}^{b} y(t)^{\delta-1} y^{\prime}(t) d t=\frac{1}{\delta} y(b)^{\delta} \\
& =\frac{1}{\delta}\left(\int_{a}^{b}\left|x^{\prime}(t)\right| P(t)^{-\frac{1}{o}} P(t)^{\frac{1}{o}} d t\right)^{\delta} \\
& =\frac{1}{\delta}\left(\int_{a}^{b} P(t)^{1-\rho} d t\right)^{\frac{\delta}{\rho}}\left(\int_{a}^{b}\left|x^{\prime}(t)\right| P(t)^{\frac{1}{\delta}} d t\right)^{\frac{\delta}{o}} .
\end{aligned}
$$

We are able to generalized inequality (2), (3) and some remarks on Opial- type inequalities.

\section{REFERENCES}

Adeagbo-Shelkh, A. G and Imoru, C. O., 2006. An Integral Inequality of the Hardy's-Type. Kragujevac J. Math. 29, 57-61.

Calvert, J., 1967. Some generalization of Opial's Inequality, Pron. Amer. Math. Soc.18, 72-75.

Maroni, P. M., 1967. Surl'ingelite d'Opial-Bessack,C.R Aca. Sci. Paris A264, 62-64.
Fabulurin, O. O., Adeagbo-Shelkh, A. G and Anthonio, Y. O., 2010. On an inequality ralates to Opial, Octogon Mathematics Magazine, 18, No 1, 32-41.

Opial, Z., 1960. Surune int $e^{\prime}$ galit $e^{\prime}$. Ann. Polon. Math. 8, 29-32. 
